7th International Workshop on Astronomy and

Relativistic Astrophysics (IWARA 2016)

International Journal of Modern Physics: Conference Series

Vol. 45 (2017) 1760060 (4 pages)

(C) The Author(s)

DOI: $10.1142 / \mathrm{S} 2010194517600606$

\title{
Neutral Pion Pole Mass Calculation in a Strong Magnetic Field: Lattice QCD Versus NJL Model
}

\author{
Ricardo L. S. Farias \\ Departamento de Física \\ Universidade Federal de Santa Maria (UFSM) \\ 97105-900 Santa Maria, RS, Brazil \\ ricardo.farias@ufsm.br \\ Sidney S. Avancini, Marcus Benghi Pinto, William R. Tavares \\ Departamento de Física \\ Universidade Federal de Santa Catarina (UFSC) \\ 88040-900 Florianópolis, Santa Catarina, Brazil \\ sidney.avancini@ufsc.br, marcus.benghi@ufsc.br,william.tavares@posgrad.ufsc.br \\ Varese S. Timóteo \\ Grupo de Óptica e Modelagem Numérica (GOMNI) \\ Faculdade de Tecnologia \\ Universidade Estadual de Campinas (UNICAMP) \\ 13083-970 Campinas, SP, Brazil \\ varese@ft.unicamp.br
}

Published 15 August 2017

\begin{abstract}
Within the framework of cold magnetized SU(2) Nambu-Jona-Lasinio model we evaluate the $\pi_{0}$ and $\sigma$ pole mass, as well as, $f_{\pi_{0}}, g_{\pi_{0} q q}$ at zero baryon density. We employ a magnetic field dependent coupling, $G(e B)$, fitted to reproduce lattice QCD results for the quark condensates. In particular, we find that the $\pi_{0}$ meson mass systematically decreases when the magnetic field increases, in good agreement with recent lattice calculations.
\end{abstract}

Keywords: Neutral Meson Mass, RPA Approximation, NJL Model in a Magnetic Field.

PACS numbers: 14.40.Aq, 12.39.-x, 12.38.-t, 13.40-f, 12.40.-y, 12.38.Gc

\section{Introduction}

In this work the $\pi_{0}$ neutral meson pole mass in a strong magnetic field is calculated within the random phase approximation (RPA) ${ }^{1}$ at zero temperature and baryon

This is an Open Access article published by World Scientific Publishing Company. It is distributed under the terms of the Creative Commons Attribution 4.0 (CC-BY) License. Further distribution of this work is permitted, provided the original work is properly cited. 


\section{R. L. S. Farias et al.}

density. The model coupling constant, $G(e B)^{2,3}$, is taken to be magnetic field dependent and it has been fitted in order to reproduce lattice QCD results for the quark condensates ${ }^{4}$. We employ the magnetic field independent regularization scheme (MFIR) ${ }^{5}$.

\section{Meson Properties Under Strong Magnetic Field}

The SU(2) NJL model lagrangian, in the presence of a magnetic field, is described by

$$
\mathcal{L}=\bar{\psi}_{f}(i \not D-\tilde{m}) \psi_{f}+G\left[\left(\bar{\psi}_{f} \psi_{f}\right)^{2}+\left(\bar{\psi}_{f} i \gamma_{5} \vec{\tau} \psi_{f}\right)^{2}\right]-\frac{1}{4} F^{\mu \nu} F_{\mu \nu}
$$

where a sum over repeated $f$ is implied. The electromagnetic gauge field is represented by $A^{\mu}, F^{\mu \nu}=\partial^{\mu} A^{\nu}-\partial^{\nu} A^{\mu}, \vec{\tau}$ is the isospin matrix, the coupling constant by $G$ while $Q=\operatorname{diag}\left(q_{u}=2 e / 3, q_{d}=-e / 3\right)$ represents the charge matrix, $D^{\mu}=\left(i \partial^{\mu}-Q A^{\mu}\right)$ is the covariant derivative, $\psi^{\mathrm{T}}=\left(\psi_{u}, \psi_{d}\right)$ is the quark fermion field and $\tilde{m}=\operatorname{diag}\left(m_{u}, m_{d}\right)$ represents the bare quark mass matrix. It has been shown by Avancini et al. ${ }^{1}$ that within the RPA approximation the $\pi_{0}$ mass can be written as:

$$
m_{\pi_{0}}^{2}(B)=-\frac{m}{M(B)} \frac{1}{4 i G N_{c} N_{f} I\left(m_{\pi_{0}}^{2}, B\right)}
$$

where

$$
I\left(m_{\pi_{0}}^{2}, B\right)=\frac{1}{4(2 \pi)^{3}} \sum_{f=u, d} \beta_{f} \sum_{n=0}^{\infty} g_{n} I_{f, n}\left(k_{\|}^{2}=m_{\pi_{0}}^{2}\right),
$$

where $M(B)$ is the effective mass, $\beta_{f}=\left|q_{f}\right| B, f=(u, d), N_{c}=3, g_{n}=2-\delta_{n 0}$, $p_{\|}=p_{0}-p_{3}$, and

$$
I_{f, n}\left(k_{\|}^{2}\right)=\int d^{2} p_{\|} \frac{1}{\left[p_{\|}^{2}-M^{2}-2 \beta_{f} n\right]\left[(p+k)_{\|}^{2}-M^{2}-2 \beta_{f} n\right]} .
$$

It can be shown ${ }^{1}$ that the $\sigma$-meson mass is given by the relation $m_{\sigma}^{2}(B)=$ $4 M^{2}(B)+m_{\pi_{0}}^{2}(B)$ and that the Gell-Mann-Oakes-Renner (GOR) relation in a magnetic medium is recovered ${ }^{1}$. The detailed calculation of $f_{\pi_{0}}$ pion decay constant and $g_{\pi_{0} q q}$ can be found elsewhere ${ }^{1}$. We use the four different sets of parameters displayed in Table 1.

Table 1. Parameter sets for the NJL model at $T=B=0$. The correct $e B \rightarrow 0$ limit of our ansatz requires that $G_{I I}=G(e B=0)$.

\begin{tabular}{ccccc}
\hline & $m_{\pi_{0}}(\mathrm{MeV})$ & $m_{0}(\mathrm{MeV})$ & $G\left(\mathrm{GeV}^{-2}\right)$ & $\Lambda(\mathrm{MeV})$ \\
\hline Set I & 135.62 & 5.0 & $G_{I}=4.67$ & 664.3 \\
Set II & 143.31 & 5.5 & $G_{I I}=4.50$ & 650.0 \\
Set III & 417 & 48.41 & $G_{I I I}=G_{I}$ & 664.3 \\
Set IV & 417 & 50.16 & $G_{I V}=G_{I I}$ & 650.0 \\
\hline
\end{tabular}



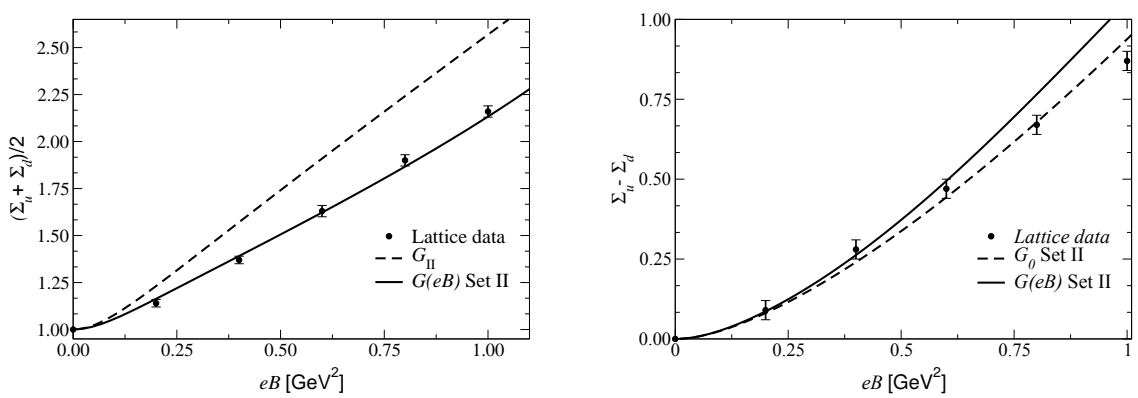

Fig. 1. Condensates average and difference as functions of $e B$ for the NJL model with $G_{I I}, G(e B)$ compared to lattice QCD calculations ${ }^{4}$.

\section{Field Dependent Coupling $G(e B)$}

Next, we present our ansatz for $G(e B)$ which reproduces the lattice results of Bali et al. ${ }^{4}$. Lattice simulations for the average $\left(\Sigma_{u}+\Sigma_{d}\right) / 2$ can be obtained by using

$$
G(e B)=\alpha+\beta e^{-\gamma(e B)^{2}},
$$

where $\alpha=1.44373 \mathrm{GeV}^{-2}, \beta=3.06 \mathrm{GeV}^{-2}$ and $\gamma=1.31 \mathrm{GeV}^{-4}$. In Fig.1 we show NJL results for the condensate average and difference using $G(e B)$ and $\mathrm{G}$, and compare with lattice results.

\section{Numerical Results and Conclusions}

The properties of magnetized neutral mesons have been investigated using a fixed and a $B$-dependent coupling constant so that model predictions and LQCD results related to inverse magnetic catalysis agree ${ }^{2,3}$. In Fig. 2 our results for the $m_{\pi_{0}}$ and $g_{\pi_{0} q q}$ are presented for both fixed $G$ and $G(e B)$. We have found that the use
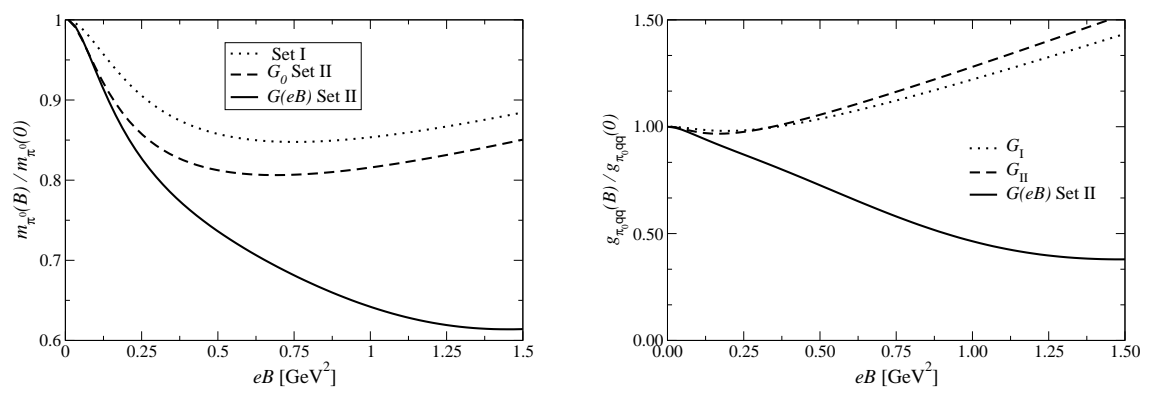

Fig. 2. Normalized $\pi_{0}$ meson mass and meson-quark coupling as a function of $e B$ in the NJL model with different coupling schemes. 


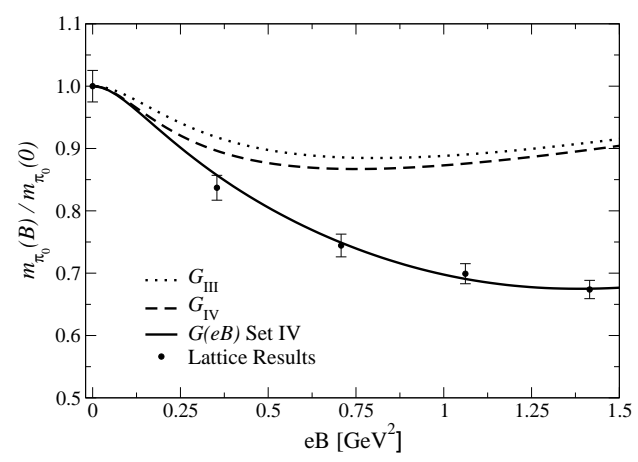

Fig. 3. Normalized neutral pion mass $m_{\pi_{0}}(e B) / m_{\pi_{0}}(0)$ in the NJL model with different coupling schemes and a large current quark mass compared to recent lattice results ${ }^{6}$.

of $G(e B)$ and $G_{I}$ (and $G_{I I}$ ) indicate an opposite behavior for $g_{\pi_{0} q q}$ with $B$. As a major result, we show that the $\pi_{0}$ remains a soft mode even at rather high field strengths $\left(\approx 1.5 \mathrm{GeV}^{2}\right)$ since its mass decreases by about $30 \%$. Our results in Fig.7 show a remarkable agreement with recent LQCD predictions ${ }^{7}$.

\section{Acknowledgements}

This work was supported by CNPq, FAPESP and CAPES.

\section{References}

1. S. S. Avancini, W. R. Tavares, and M. B. Pinto, Phys. Rev. D 93, 014010 (2016).

2. R. L. S. Farias, K. P. Gomes, G. Krein, and M. B. Pinto, Phys. Rev. C 90, 025203 (2014).

3. R. L. S. Farias, V. S. Timoteo, S. S. Avancini, M. B. Pinto, and G. Krein, arXiv:1603.03847 [hep-ph](2016).

4. G. S. Bali, F. Bruckmann, G. Endrödi, Z. Fodor, S. D. Katz, S. Krieg, and A. Schäfer, Phys. Rev. D 86, 071502(R) (2012).

5. D. P. Menezes, M. B. Pinto, S. S. Avancini, A. Pérez Martínez, and C. Providência, Phys. Rev. C 79, 035807, (2009); P. G. Allen, A. G. Grunfeld, and N. N. Scoccola, Phys. Rev. D 92, 074041, (2015); D. C. Duarte, P. G. Allen, R. L. S. Farias, P. H. A. Manso, R.O. Ramos, and N. N. Scoccola, Phys. Rev. D 93, 025017 (2016).

6. G. Bali, B.B. Brandt, G. Endrödi, and B. Glaessle, PoS LATTICE2015, arXiv:1510.03899 [hep-lat](2015).

7. S.S. Avancini, R.L.S. Farias, M.B. Pinto, W.R. Tavares, and V.S. Timóteo, Phys. Lett. B 767, 247 (2017). 